\title{
El Síndrome de Kraepelin-Bleuler-Schneider y la Conciencia Moderna: Una Aproximación a la Historia de la Esquizofrenia
}

\section{Kraepelin-Bleuler-Schneider Syndrome from a Modern Perspective: An Assessment of the History of Schizophrenia}

\author{
Enric J. Novella y Rafael Huertas* \\ Centro de Ciencias Humanas y Sociales (CSIC), Madrid-España
}

\begin{abstract}
Resumen. El presente artículo propone un recorrido por la historia de la esquizofrenia en el que se describe, en primer lugar, el proceso de fijación del concepto clínico convencional en las obras de Emil Kraepelin, Eugen Bleuler y Kurt Schneider. Posteriormente, y como contrapunto a su caracterización externa u objetiva, se exponen algunas líneas maestras de la reconstrucción de la experiencia esquizofrénica acometida por la psicopatología de inspiración fenomenológica. Y, por último, se discuten los principales factores y ámbitos implicados en la constitución de la esquizofrenia como un trastorno característicamente moderno de la subjetividad que destaca por aunar anomalías de la conciencia, la vivencia del cuerpo y la vida social.
\end{abstract}

Palabras clave: Esquizofrenia, historia, subjetividad, modernidad.

\begin{abstract}
This article offers a panoramic assessment of the history of schizophrenia. It describes first the foundations of the conventional clinical concept as in the classic works of Emil Kraepelin, Eugen Bleuler and Kurt Schneider. Afterwards, and as a counterpoint to its external or objective characterization, the article presents some of the most notable aspects of the schizophrenic experiential world as reconstructed by phenomenological psychopathology. Finally, it provides a discussion of the major factors and areas involved in the constitution of schizophrenia as a typically modern disorder of subjectivity that combines disturbances of self-consciousness, abnormal bodily experiences and a severe impairment of social life.
\end{abstract}

Key words: Schizophrenia, history, subjectivity, modernity.

"Uno estaría tentado a hablar de una afinidad particular entre la histeria y el espíritu reinante antes del siglo XVIII, afinidad que existiría entre la esquizofrenia y el espíritu de nuestro tiempo”.

Karl Jaspers, Genio y locura (1922)
La correspondencia sobre este artículo puede enviarse al primer autor a: c/ Albasanz 26-28, 28037 Madrid (España). Correo electrónico: enric.novella@cchs.csic.es
"A veces cuando estoy con Maya me parece que siento la eterna juventud de las cosas, pero cuando pienso en ello esa sensación se desvanece”.

Claire Fisher en A dos metros bajo tierra (2004) 


\section{Introducción}

La esquizofrenia no es un trastorno o, si se quiere, una enfermedad mental como las demás. Implicando y comprometiendo las estructuras elementales de todo aquello que asociamos con aspectos nucleares de nuestra cultura como la individualidad, la privacidad, la identidad o la agencia, la esquizofrenia no es, como se sabe, un proceso que se padece de forma puntual, sino una condición que impregna la totalidad de la existencia y confiere a su portador un perfil psicológico constante y muy definido (Colodrón, 2002); no se tiene esquizofrenia, se es esquizofrénico (Estroff, 1989). Pero, en un sentido más amplio y atendiendo al enorme potencial simbólico que ha ido atesorando a lo largo del siglo $\mathrm{XX}$, la esquizofrenia tampoco es una enfermedad convencional, pues -como bien advirtió Jaspers- su propia naturaleza parece decirnos, sugerirnos o incluso mostrarnos algo importante y esencial con respecto al "espíritu de nuestro tiempo". De hecho, todavía hoy vemos en ella una experiencia radical que, como nuestra época, remite a la inseguridad y al miedo, a lo anómico y heterodoxo, a lo oscuro e inefable, y que conduce a una destrucción de los vínculos interpersonales, a una ruptura con la historia y la tradición y a un abandono del territorio del lenguaje y los significados compartidos. En realidad, es difícil encontrar descripciones o diagnósticos de nuestro tiempo y nuestra cultura -eso que, genéricamente, solemos denominar Modernidadque no permitan trazar paralelismos y analogías más o menos estrechas con la condición esquizofrénica (Sass, 1992; Leferink, 1997).

Partiendo de la consideración de esta afinidad constitutiva, el presente artículo propone un recorrido por la historia de la esquizofrenia en el que se intenta desentrañar su configuración no sólo como uno de los cuadros clínicos más emblemáticos de la psiquiatría contemporánea, sino como una severa perturbación de la conciencia del yo, la vivencia del cuerpo y la vida social que hunde sus raíces en el potencial de alienación que comportan las estructuras de la subjetividad moderna. Con este objetivo describimos, en primer lugar, el proceso de fijación del concepto clínico de esquizofrenia en las obras clásicas de Emil Kraepelin, Eugen Bleuler y Kurt
Schneider. Posteriormente, y como contrapunto a su caracterización externa u objetiva, exponemos algunas líneas maestras de la reconstrucción de la experiencia esquizofrénica acometida por la psicopatología de inspiración fenomenológica. Y, por último, discutimos brevemente los principales factores y ámbitos implicados en la constitución de la esquizofrenia como un trastorno estrechamente vinculado al paulatino despliegue de la individualidad reflexiva característica de nuestra cultura.

\section{De la demencia precoz a la esquizofrenia}

Como es sabido, la historia del concepto de esquizofrenia tiene su punto de partida más conocido y definido en la descripción de la llamada Dementia praecox por parte del psiquiatra alemán Emil Kraepelin (1856-1926). Sin embargo, la prioridad de esta descripción ha suscitado alguna controversia historiográfica, pues la aparición del término demencia precoz se remonta al alienismo francés de los años centrales del siglo XIX. En sus Études cliniques de 1852-53, Benedict Augustin Morel (18091873 ) presentó algunos casos de jóvenes alienados a los que diagnosticó de démence stupide o stupidité aboutisant à la démence, y que se caracterizaban por presentar una marcada estereotipia de actitudes, gestos y lenguaje, así como un negativismo muy evidente; dicho cuadro evolucionaba inexorable y rápidamente hacia una pérdida de las facultades mentales $\mathrm{y}$, en definitiva, hacia la demencia (Mahieu, 2004). Pero no fue hasta 1860 , en su Traité des maladies mentales -un intento de aplicación a la práctica psiquiátrica de la teoría de la degeneración-, cuando Morel utilizó por primera vez el término démence précoce para referir la siguiente observación:

"Un padre desafortunado me consultó un día sobre el estado mental de su hijo de 13 o 14 años de edad. [...] Una especie de torpeza próxima al embrutecimiento (hébétement) había reemplazado su actividad habitual y, cuando lo volví a ver, juzgué que la transición fatal al estado de demencia precoz estaba a punto de operarse [...]. Tal es, en muchos casos, la funesta terminación de la locura hereditaria. Una inmovilización súbita de todas las facultades, una demencia precoz, indican que el joven sujeto ha alcanzado el término de su vida intelectual” (Morel, 1860, pp. 564-565). 
El término moreliano de demencia precoz, por tanto, hay que entenderlo en el marco del más estricto degeneracionismo y, de hecho, cuando Morel describía este tipo de pacientes no estaba aislando una entidad nosológica discreta, sino explicando algunas formas características de las locuras hereditarias (Huertas, 1987). En concreto, la démence précoce constituía un ejemplo de la tercera clase de alienaciones hereditarias, esto es, de las "locuras hereditarias con una existencia intelectual limitada" (Morel, 1860 , p. 562).

Años más tarde, Kraepelin propuso el término Dementia praecox con un horizonte conceptual mucho más ambicioso, al pretender establecer una entidad nosológica diferenciada con respecto a otras enfermedades mentales y a los estados psicopáticos degenerativos (Hoenig, 1983; Beer, 1996). Aunque la homonimia ha dado lugar a algunas interpretaciones que postulan una relación genealógica más o menos laxa entre Morel y Kraepelin (Baruk, 1974; Chandrasena, 1983), parece evidente que este último no se limitó a latinizar el término moreliano y que, como bien ha indicado José María Álvarez, no existe una correspondencia clara "entre los apuntes deshilvanados de Morel y la descripción sistemática de Kraepelin" (Álvarez, 2008, p. 349). En realidad, a poco que se indague sobre el caudal de influencias que inspiraron la formulación de la Dementia praecox resulta fácil descartar una relación lineal y unívoca entre ambos autores, pues, junto al término moreliano, en la obra de Kraepelin cabe advertir también el rastro de la idea de predisposición de Valentin Magnan (1835-1916), de las nociones de endógeno y exógeno de Paul Moebius (1853-1907) o de la afinidad con el método experimental de Wilhelm Wundt (1832-1920) (Colodrón, 1983; Hoff, 1994).

Con todo, no cabe duda que fueron las descripciones de Ewald Hecker (1843-1909) de la llamada hebefrenia y de Karl Ludwig Kahlbaum (18281899) de la catatonía las que permitieron a Kraepelin reunir bajo un mismo epígrafe de "procesos de deterioro" (Verblödungsprocesse) un conjunto de cuadros clínicos formado por la Dementia praecox -más próxima a la hebefrenia de Hecker que a la démence précoce de Morel-, la Katatonie y la Dementia paranoides. Estas tres formas, agrupa- das en la $5^{\mathrm{a}}$ edición de su Lehrbuch (1896), fueron finalmente consideradas una sola enfermedad -denominada justamente Dementia praecox- a partir de la $6^{\text {a }}$ edición de 1899. Como es sabido, Kraepelin estableció entonces dos grandes grupos de psicosis endógenas: la psicosis maniaco-depresiva, curable, y la demencia precoz, incurable (Angst, 2002). Posteriormente, completó esta clasificación diferenciando dos formas evolutivas de la demencia precoz: la progresiva, que conduciría a un deterioro permanente, y la que cursaba con brotes y sin un déficit irreversible. Con el tiempo, otra de las novedades introducidas por el psiquiatra alemán fue el paulatino "arrinconamiento" de la paranoia, hipertrofiando las "formas paranoides" de la Dementia praecox y denominando parafrenias a los mismos casos que con anterioridad había etiquetado de "formas fantásticas de la paranoia" (Álvarez, 1996). Aunque la agrupación en una misma enfermedad de estas formas paranoides con la hebefrenia y la catatonía resultaba algo forzada desde el punto de vista teórico y fue muy criticada por los psiquiatras franceses (Séglas, 1900; Serbski, 1903-04), Kraepelin creía que se trataba de "un conjunto de cuadros clínicos que tienen la particularidad común de conducir a estados de un deterioro psíquico característico" (Kraepelin, 1899, p. 167). Y, de este modo, instituyó toda una serie de síntomas deficitarios -deterioro afectivo (gemüthliche Verblödung), apatía, indiferencia, desorganización del pensamiento, disgregación psíquica (Zerfahrenheit), etc.- como las manifestaciones nucleares de la Dementia praecox, a las que se asociaban un cortejo variable de síntomas accesorios como ideas delirantes, alucinaciones o trastornos psicomotores.

La concepción kraepeliniana de la Dementia praecox tuvo una amplia difusión en las primeras décadas del siglo XX, aunque los procedimientos de su artífice, basados en criterios supuestamente objetivos como la etiopatogenia, la clínica y la evolución resultaron pronto excesivamente rígidos para el desarrollo del conocimiento psiquiátrico (LantériLaura, 2000). Así las cosas, en 1911 el psiquiatra suizo Eugen Bleuler (1857-1940) publicó su Dementia praecox oder Gruppe der Schizophrenien, donde ofrecía una caracterización del cuadro no a partir de su evolución, sino de lo que consideraba su 
rasgo psicopatológico fundamental: la escisión del yo. Esta nueva perspectiva llevó a Bleuler a introducir el neologismo "esquizofrenia" (literalmente, "mente escindida") para denominar la entidad aislada por Kraepelin, pero su propuesta no se limitaba a sugerir una mera variación terminológica, sino que se derivaba de una nueva mirada al loco y su locura en la que la clínica -sintomatología y evoluciónpasaba a un segundo plano y la interpretación psicopatológica asumía una importancia central (Gruhle, 1913)

En este sentido, resulta particularmente interesante señalar las notables diferencias existentes entre el proceder, las influencias y, muy especialmente, el contexto de observación en que Kraepelin y Bleuler realizaron sus aportaciones. El primero estaba en Heidelberg cuando vieron la luz la $4^{\mathrm{a}}$, la $5^{\text {a }}$ y la $6^{\text {a }}$ edición de su Lehrbuch, pero las ediciones anteriores aparecieron durante su estancia en la clínica psiquiátrica de la Universidad de Dorpat (Estonia), donde había ejercido entre 1886 y 1890. La barrera idiomática existente entre el médico alemán y la mayoría de sus pacientes, que sólo hablaban dialectos eslavos, debió desempeñar un papel importante en su método de exploración, pues limitó ostensiblemente el interés de Kraepelin por los testimonios de unos pacientes a los que apenas comprendía. Por el contrario, Bleuler había ocupado la dirección del Hospital suizo de Rheinau entre 1886 y 1898, antes de asumir la cátedra de psiquiatría en su Zurich natal y la dirección de la clínica universitaria de Burghölzli. Su trato con los pacientes fue así muy distinto, ya que no sólo pudo comunicarse en su propia lengua, sino que se afanó en establecer con ellos un contacto afectivo y un vínculo personal muy cercano (Ellenberger, 1976). Por lo demás, Bleuler acusó la influencia de toda una serie de autores como Wilhelm Griesinger (18171869), Jean-Martín Charcot (1825-1893), Sigmund Freud (1856-1939) o Pierre Janet (1859-1947) que le animaron a cultivar el análisis psicológico de la enfermedad mental, a pesar de que -también en el caso de la esquizofrenia- nunca dejó de tener por segura la intervención de causas orgánicas en su desencadenamiento y dinámica inicial (Bleuler, 1926; Bovet y Seywert, 1995; Stotz-Ingen-lath, 2000).
Pues bien, en estas coordenadas clínicas y científicas, Bleuler revisó la obra de Kraepelin y "descubrió" una serie de "síntomas fundamentales" que, como ha señalado Jean Garrabé, "no constituyen una sintomatología propiamente dicha, es decir, un conjunto de fenómenos que se pueda describir u observar directamente en la clínica, sino más bien una hermenéutica, la interpretación psicopatológica que se puede dar a estos fenómenos" (Garrabé, 1996, p. 57). Estos síntomas fundamentales (las célebres cuatro A de Bleuler) incluían, por este orden, trastornos de las asociaciones (los pacientes operan con ideas y conceptos que no tienen relación y pierden su continuidad, produciendo asociaciones "ilógicas" y formalmente incoherentes), trastornos afectivos (con gran tendencia al retraimiento y aplanamiento emocional o a la aparición de una afectividad incongruente), ambivalencia (presencia simultánea de pensamientos, sentimientos o actitudes contradictorias) y autismo (una manifestación particular y característica consistente en el predominio morboso de la vida interior sobre la vida de relación). A su juicio, dichos síntomas eran los constantes y exclusivos del esquizofrénico, que podría presentar además otros más llamativos pero "accesorios" como delirios, alucinaciones, perturbaciones de la memoria o trastornos del lenguaje. Asimismo, y con el fin de "comprender la enfermedad esquizofrénica", Bleuler propuso distinguir los síntomas surgidos directamente del "proceso patológico" (los "síntomas primarios") de aquellos "síntomas secundarios que sólo comienzan a operar cuando la psique enferma reacciona" (Bleuler, 1961, p. 361). Y, por último, estableció un total de cinco formas clínicas, coincidiendo en tres de ellas con las formas kraepelinianas (subgrupos paranoide, catatónico y hebefrénico) y añadiendo una forma simple (caracterizada por presentar únicamente los síntomas fundamentales) y otra "latente", compensada o paucisintomática.

En cualquier caso, lo que en su opinión definía el proceso esquizofrénico era, como ya hemos señalado, una "escisión" (Spaltung) de las funciones psíquicas resultante de la acción de "complejos" independientes o cadenas de representaciones con un determinado tono afectivo que, al dominar sucesivamente la personalidad, comprometían su unidad. 
Inspirado en los estudios psicométricos de su asistente Carl Gustav Jung (1875-1961) sobre los trastornos de las asociaciones de ideas (Jung, 1906) y en las primeras explicaciones de Freud sobre los mecanismos rectores de las formaciones inconscientes, Bleuler creía que en la esquizofrenia actuaba una debilidad asociativa primaria que impedía una integración adecuada de los contenidos de la conciencia y conducía así a la desorganización del psiquismo característica de la enfermedad. Y, de este modo, pudo proponer -si bien de una forma muy imprecisa- una suerte de trastorno generador de sus manifestaciones psicopatológicas que, como han recordado algunos autores, guarda una gran semejanza con los mecanismos disociativos descritos por Janet en relación con la histeria (LeBlanc, 2001).

En este punto, no podemos dejar de valorar, aunque sea muy brevemente, la deuda de Bleuler con Sigmund Freud y el psicoanálisis, que -como era de esperar- ha sido objeto de apreciaciones divergentes. Así, si para algunos autores "el punto de partida y la originalidad de las concepciones de Bleuler se deben a su enfoque psicoanalítico de los síntomas esquizofrénicos" (Bercherie, 1986, p. 154), para otros dicha influencia resulta más difícil de establecer, pues la impronta psicoanalítica le llegó a Bleuler a través de Jung y estuvo muy matizada por la psicología asociacionista de Wundt y Theodor Ziehen (1862-1950) (Colodrón, 1983). Sea como fuere, y aunque la influencia del psicoanálisis desempeñó un papel importante en la elaboración inicial del concepto de esquizofrenia, lo cierto es que la doctrina de Bleuler se alejó paulatinamente de los principios freudianos y, de hecho, en 1926 presentó en el Congreso de Alienistas en Lengua Francesa una ponencia en la que reconocía ser "un adepto a las ideas de Freud", pero -añadía- "no puedo admitir su teoría de la evolución del instinto sexual y aún menos la del origen puramente psicógeno de la esquizofrenia" (Bleuler, 1996, p. 675).

Así pues, y a pesar de que con el tiempo Bleuler fue asumiendo una visión más restrictiva de la esquizofrenia como una única enfermedad (y no un grupo de trastornos como sugería el título de su monografía de 1911) de base orgánica, la categoría asumió en sus manos una amplitud mucho mayor que la de la demencia precoz kraepeliniana, de modo que una de las consecuencias más notables de su obra fue justamente su extensión a la práctica totalidad de los cuadros psicóticos y, por tanto, una problemática generalización de su uso (Hoenig, 1983). En esas circunstancias, el empeño de los psiquiatras alemanes se diversificó en las décadas siguientes entre el análisis exhaustivo de la psicopatología específicamente esquizofrénica -esfuerzo que culminaría en un importante y hoy poco conocido volumen colectivo aparecido en 1932 (Wilmanns, 1932)- y la identificación clínica de aquellos signos o síntomas que permitieran un diagnóstico más circunscrito y fiable. En este sentido, el autor que se distinguió de un modo más notable fue, sin duda, Kurt Schneider (1887-1967), que asumió en 1946 la prestigiosa cátedra de Heidelberg y proporcionó una influyente caracterización de los síntomas esquizofrénicos. Eludiendo toda referencia a la evolución o al pronóstico y creyendo que la mera observación clínica (esto es, desprovista de cualquier consideración teórica) era suficiente para establecer el diagnóstico, Schneider invirtió la jerarquía entre los síntomas fundamentales y accesorios de Bleuler y aisló una serie de experiencias psicóticas que denominó de "primer rango" por su utilidad para detectar la presencia de lo esquizofrénico. Tal como formuló a partir de 1950 en su Psicopatología clínica, estos síntomas incluían "la sonorización del pensamiento, la audición de voces que opinan y replican al sujeto, la audición de voces que comentan sus acciones, las experiencias corporales de influencia, el robo del pensamiento y otras influencias ejercidas sobre el pensamiento, la difusión del pensamiento, la percepción delirante y la convicción de ser influenciado en los sentimientos, tendencias y voliciones" (Schneider, 1997, p. 171).

A pesar de su deseo de mantenerse en los márgenes estrictos de la clínica, Schneider no pudo evitar reconocer que la mayoría de los síntomas de primer rango compartían un rasgo fenomenológico común, y que éste consistía en una pérdida de los límites del yo o un aumento mórbido de la permeabilidad en la barrera entre el yo y el mundo. Más concretamente, los síntomas de primer rango destacaban justamente por abarcar todas las manifestaciones de lo que los alienistas 
franceses habían definido como "xenopatía", esto es, la experiencia de la propia actividad psíquica (lenguaje, pensamientos, sentimientos, intenciones, etc.) como ajena o impuesta. Y, de este modo, la aportación de Schneider suministró el tercer gran pilar sobre el que sigue descansando en la actualidad el concepto de esquizofrenia (Andreasen, 1997; Peralta y Cuesta, 2000), añadiendo al deterioro kraepeliniano y a la desorganización bleuleriana los llamados "trastornos del yo" (Ich-Störungen) como eje central de sus manifestaciones clínicas (Ramos, 1992).

\section{La experiencia esquizofrénica}

A mediados del siglo XX, pues, los componentes esenciales del concepto clínico de esquizofrenia habían quedado medianamente establecidos sobre la base de las obras de Kraepelin, Bleuler y Schneider. De hecho, a pesar de la intensa actividad investigadora desarrollada en este campo y de algunas propuestas muy difundidas como la distinción entre esquizofrenia positiva y negativa (Andreasen, 1982) o los dos síndromes de Timothy J. Crow (Crow, 1985), el diagnóstico se sigue realizando hoy en día recurriendo a una amalgama de criterios operacionales inspirados en sus descripciones (Maj, 1998). En realidad, ni la introducción de los neurolépticos a partir de la década de 1950, ni la aplicación de técnicas de neuroimagen, ni los estudios genéticos que han venido completándose desde entonces han dado lugar a modificaciones sustanciales en este sentido (Colodrón, 2002), y la esquizofrenia continúa siendo una noción eminentemente clínica, de contornos difusos y cuyos correlatos neurobiológicos distan de poder ser identificados de forma concluyente. Por ese motivo, y teniendo en cuenta las implicaciones pronósticas y el alto poder estigmatizante del término, su utilidad (Brockington, 1992) o incluso su misma validez (Boyle, 1990) son puestas en duda periódicamente. Pero, con todo, no cabe duda de que, a pesar de su naturaleza elusiva, la condición esquizofrénica constituye una forma distintiva de alienación que imprime un sello muy particular al conjunto de la experiencia del individuo.

Inicialmente descartado por el propio Jaspers con su célebre dogma de la incomprensibilidad de las vivencias psicóticas (Jaspers, 1946), el análisis del mundo interno y la subjetividad esquizofrénica se cuentan entre las grandes aportaciones de la psicopatología de inspiración fenomenológica (Kraus, 2001). Con el doble objetivo de lograr una comprensión global del cuadro clínico y de reconstruir "desde dentro" su experiencia, las descripciones y reflexiones legadas desde esta tradición han logrado aproximarse a una caracterización de la alienación esquizofrénica que no sólo dota de inteligibilidad a sus distintas manifestaciones clínicas, sino que permite ubicarla y entenderla en unas coordenadas históricas y culturales más amplias. En líneas generales, el análisis fenomenológico se ha centrado en dar cuenta de tres órdenes de fenómenos que conciernen, respectivamente, a la vida social, a la vivencia del cuerpo y a los propios contenidos de la conciencia. Y así, dependiendo del marco teórico de referencia y de la relevancia otorgada a cada uno de estos fenómenos, se han propuesto distintas interpretaciones que, a pesar de sus divergencias, coinciden en una serie de aspectos y puntos esenciales.

La primera concepción de la esquizofrenia que cabe destacar en este sentido fue ofrecida en 1927 por el psiquiatra franco-polaco Eugène Minkowski (1885-1972). Inspirándose en la obra de Henri Bergson (1859-1941), Minkowski tomó el autismo como el trastorno fundamental de la enfermedad, y lo definió como una "pérdida del contacto vital con la realidad" (Minkowski, 2000, p. 88). Partiendo de la oposición bergsoniana entre el instinto y la inteligencia, Minkowski pensaba que la esquizofrenia, a diferencia de otras formas de locura caracterizadas por el deterioro intelectual, implicaba antes que nada un "déficit pragmático" y una alteración del dinamismo mental derivada de un debilitamiento del "instinto vital" (élan vital). De este modo, decía, se producía una pérdida de

“esa fibra sensible que nos permite vibrar al unísono con nuestros semejantes, que nos vincula con la personalidad íntima de cada uno de ellos, que permite compenetrarnos con ella y sentirnos uno con ella. El contacto personal está roto, la mirada ya no sabe fijarse en las personas del entorno en la medida en que la vida lo exige de nosotros, resbala de inmediato por encima y parte hacia las regiones desérticas y glaciales regidas por la inteligencia pura" (Minkowski, 2000, p. 111). 
En una de sus formulaciones más conocidas, Minkowski denominó "racionalismo mórbido" esta tendencia esquizofrénica al solipsismo, la abstracción y el esprit de géometrie que llevaba a los pacientes a excluir de su psiquismo "todo lo que es irracional, todo lo que es cambio, todo lo que es progresión" y, en definitiva, "todo lo que constituye la riqueza y la movilidad de la vida" (Minkowski, 2000 , p. 115). El racionalismo mórbido, por tanto, constituía un fenómeno complementario del autismo en tanto en cuanto ambos resultaban de una falla en la conexión intuitiva, espontánea o prerreflexiva con el mundo y con los demás; pero, asimismo, ambos fenómenos solían acompañarse de una "cenestesia aberrante", esto es, de una profunda alienación con respecto a la propia vitalidad, los afectos o las sensaciones de carácter orgánico. Nada como el testimonio de uno de sus pacientes parecía condensar mejor la naturaleza, las consecuencias y la radicalidad de todos estos desórdenes:

"Suprimí la afectividad, como lo hice con toda la realidad. Existo desde el punto de vista cuerpo, pero no tengo ninguna sensación interna de la vida. Ya no siento las cosas. Ya no tengo sensaciones normales. Suplo esa falta de sensaciones normales por la razón” (Minkowski, 2000, pp. 118-119).

Fuera de los círculos de la psiquiatría francesa, la obra de Minkowski fue poco conocida hasta la década de 1960, cuando fue abiertamente elogiada e incluso imitada por Ronald D. Laing y el grupo de los antipsiquiatras ingleses (Laing, 1964). Pero, a pesar de desconocer, soslayar o rechazar los conceptos bergsonianos que le habían servido de apoyo, otros autores llegaron a conclusiones muy similares desde referentes teóricos muy distintos. Así, por ejemplo, el psiquiatra holandés Henrik C. Rümke (1893-1967) describió en 1941 el "sentimiento precoz" como la inefable sensación de vacío inducida en el observador por el autismo esquizofrénico, que él consideraba un elemento diagnóstico de primer orden y un fenómeno derivado de una debilidad específica del "instinto de acercamiento" y, en suma, de un agotamiento de la "fuente de energía psíquica" provocado por la enfermedad (Rümke, 1990, p. 339).

Por su parte, y tomando como punto de partida la obra tardía de Edmund Husserl (1859-1938), el alemán Wolfgang Blankenburg (1928-2002) publicó en
1971 Der Verlust der natürlichen Selbstverständlichkeit [La pérdida de la evidencia natural], donde ofreció una importante aportación a la psicopatología fenomenológica sobre la que merece la pena detenerse algo más. Blankenburg se sirvió en ella del concepto de sentido común como elemento vertebrador de una propuesta centrada en el análisis de la esquizofrenia paucisintomática, es decir, con pocos síntomas, desde el punto de vista de las anomalías de la intersubjetividad y la corporalidad. En su opinión, este concepto de origen clásico reunía al menos tres significados clave que hacían de él un excelente organizador en la comprensión fenomenológica de la esquizofrenia. En tanto que cenestesia, esto es, en su acepción naturalista, el sentido común es la facultad que posibilita la síntesis funcional de todas las sensaciones particulares y dota a la percepción de unidad y coherencia, formando así la base de la autoconciencia (Fuchs, 1995). En la acepción humanista de procedencia latina, el sensus communis alude a esa suerte de conocimiento práctico (en parte equivalente a la phrónesis de los griegos) que nos permite concebir objetos, situaciones o la conducta de otros desde un horizonte compartido de sentido o, dicho en los términos empleados por Blankenburg, desde una "evidencia natural" o prerreflexiva (Blankenburg, 1971). Y, por último, el sentido común apunta también a la capacidad emocional y cognitiva de conectar y acceder intuitivamente al mundo interno del otro y establecer aquello que el mismo Bleuler definió como sintonía (Bleuler, 1969). Por medio de este concepto, pues, la autoconciencia sensorial y la percepción integrada del mundo físico y de uno mismo aparecen estrechamente vinculadas con la facultad de aprehender e interpretar adecuadamente las acciones de otros en la esfera social, pues ésta no es sino el resultado de una capacidad de resonancia intercorporal que presupone un "habitar" con plenitud el propio cuerpo y, en definitiva, un sentirse uno mismo como el sujeto de sus percepciones, sensaciones, pensamientos o actos. Y, de este modo, la radicalidad de la alienación esquizofrénica se derivaría del hecho de implicar una suerte de liberación del psiquismo de su anclaje corporal, de manera que, como ha sugerido recientemente el psiquiatra italiano Giovanni Stanghellini, la persona sólo puede experimentarse 
como si de las substancias cartesianas se tratase, esto es, como un "espíritu desencarnado" o un "cuerpo inanimado" (Stanghellini, 2004).

En los últimos años, y bajo la influencia del pensamiento de Maurice Merleau-Ponty (1908-1961) y la distinción entre cuerpo vivido y cuerpo anatómico (o entre cuerpo-sujeto y cuerpo-objeto), esta visión de la esquizofrenia como un "desencarnamiento del yo" goza de un amplio predicamento en la literatura fenomenológica (Fuchs, 2005). Sin embargo, otras interpretaciones recientes han vuelto a poner el punto de mira en la especificidad de la conciencia esquizofrénica como la instancia fundamental desde la que entender la naturaleza última de sus perturbaciones y la aparente disparidad de sus manifestaciones clínicas. Este es el caso, muy especialmente, del psicólogo norteamericano Louis A. Sass, que ha insistido en diversas publicaciones en considerar la esquizofrenia como un trastorno de la conciencia del yo o la autoconciencia prerreflexiva -que Sass, siguiendo al filósofo francés Michel Henry, prefiere denominar ipseidad- caracterizado por dos tipos de distorsiones básicas pero complementarias de la actividad psíquica: la "hiperreflexividad" y la disminución de la "autoafección" (Sass, 1992; Sass y Parnas, 2003). Por hiperreflexividad Sass entiende una forma exagerada y aberrante de autoconciencia que provoca que los sucesos mentales, interiores o subjetivos sean experimentados de forma similar a los objetos del mundo físico. De este modo, por ejemplo, las alucinaciones verbales características de la esquizofrenia consistirían en una ruptura del silencio habitual con que se desarrolla nuestro incesante diálogo interno debido a esa objetivación mórbida que sufren los contenidos de la conciencia hiperreflexiva (Stanghellini y Cutting, 2003; Álvarez y Colina, 2007). Por su parte, la disminución de la autoafección implica un debilitamiento de la autodisposición del sujeto y de la vivencia prerreflexiva de presencia o existencia como fuente de vida que conlleva, como ya hemos visto, una pérdida de la sensación yoica que impregna todos nuestros pensamientos, percepciones y actos y, en consecuencia, una notoria alienación con respecto a ellos. Por lo demás, estos dos tipos de distorsiones se acompañan de una serie de anomalías perceptivas derivadas de un desplazamiento en la relación focal-tácito o explícito-implícito, de manera que el esquizofrénico tiende a percibir como explícitos elementos del campo perceptivo que habitualmente suelen permanecer implícitos (y de ahí, por ejemplo, sus frecuentes síntomas hipocondríacos) o es incapaz de acceder a esa "dimensión tácita" (Polanyi, 1967) en que mayormente se desenvuelve nuestra vida psíquica y de relación.

En síntesis, puede decirse que, con todas las diferencias teóricas de partida o de énfasis en los trastornos de la vida social, la vivencia del cuerpo o la conciencia del yo, la mirada fenomenológica ha permitido perfilar los contornos de la experiencia esquizofrénica en unos términos muy alejados de la visión tradicional de la locura como una regresión o claudicación "incomprensible" de la razón. De hecho, se ponga el acento en el autismo, la obliteración del sentido común o las distorsiones de la ipseidad, lo cierto es que el mundo esquizofrénico se revela más bien como un complejo universo similar al representado por algunos pintores de vanguardia como Giorgio de Chirico o René Magritte: solitario, cerebral, glacial, y perfectamente adinámico e inerte. Y, en este sentido, no sorprende que la experiencia esquizofrénica haya sido equiparada a menudo con la misma conciencia moderna y su ciencia positivista (Sass, 1992; Stanghellini, 2004), pues ambas tienden a desencarnar el mundo y sustraerle todo aliento, vitalidad o trascendencia; desplazan al sujeto más allá de un orden compartido de sentido; reducen los fenómenos a su mera apariencia y transforman la vida en un simple mecanismo del que, en última instancia, sólo cabe ser un espectador desapasionado.

\section{La cultura de la esquizofrenia}

La mención de estas analogías de la esquizofrenia con la conciencia moderna nos conduce finalmente a plantear algunas consideraciones sobre la afinidad constitutiva entre ambas y, más genéricamente, sobre los principales factores y ámbitos implicados en la emergencia de la esquizofrenia como un acontecimiento estrechamente relacionado con el desarrollo de la cultura moderna. En este punto, conviene advertir que asumir esta perspectiva no implica 
postular una relación de causalidad unívoca y directa entre la esquizofrenia y la Modernidad occidental, pues, como es sabido, esta relación no ha sido confirmada -y sí desmentida en diversas ocasiones- por medio de estudios transculturales comparativos (Fabrega, 1989). Pero, dado que apenas existen descripciones claramente compatibles con el síndrome nuclear de la esquizofrenia anteriores a las primeras décadas del siglo XIX (Fraguas y Breathnach, 2009) y que su misma formulación clínica se remonta al tránsito del siglo XIX al XX, parece innegable que el despliegue de la Modernidad ha participado de un modo determinante y decisivo en su constitución como "objeto cultural" (esto es, en "algo" para "alguien") (Leferink, 1997).

Así, y del mismo modo que la esquizofrenia no afecta al individuo antes de la adolescencia -ya que sólo en este periodo de la vida parecen emerger las condiciones psíquicas para ello (Arieti, 1965)-, también a nivel social y cultural ha sido necesario que concurrieran una serie de circunstancias para que un patrón previamente indefinido de alteridad y subjetividad pudiera ser concebido e identificado como "esquizofrénico". Y, teniendo en cuenta que, como hemos visto, la esquizofrenia compromete elementos cardinales de nuestro orden social y cultural como la atribución de agencia psicológica, la construcción de la identidad, el desempeño secuencial de roles sociales o la presentación pública del yo, no resulta difícil aislar algunos factores que, como ahora mismo veremos, confluyen en dos puntos básicos, a saber, la espectacular movilización social y psicológica inducida en los individuos por el proceso de modernización y el paulatino despliegue de la individualidad reflexiva característica de nuestra cultura.

Ciertamente, el primer factor concierne a uno de los descriptores preferentes con los que la propia Modernidad ha tendido a entenderse a sí misma. No en vano, la Modernidad ha sido definida a menudo como una suerte de "movilización general" que ha conducido a mutaciones decisivas en la experiencia del tiempo y el espacio, los estilos de pensamiento y acción, las pautas de conducta social (marcadas ahora por un notable incremento e intercambio de roles) o las relaciones entre generaciones, sexos y clases (Giddens, 1990). A estos cambios habría que añadir la amplia movilización producida en el plano cultural, sobre todo en la forma de una ruptura individualista con la tradición y un pluralismo ideológico y religioso que, aun reconociendo todo su potencial emancipador, ha provocado que los sistemas de valores y las reservas de sentido hayan dejado de ser un patrimonio común de todos los miembros de la sociedad (Berger y Luckman, 1997). Como consecuencia de todos estos procesos y de un vertiginoso desarrollo tecnológico, la Modernidad ha forjado una profunda transformación en el estilo y el ritmo de vida que habitualmente solemos describir recurriendo a expresiones como "dinamismo", "aceleración" o "inquietud" (véase, por ejemplo, el mismo Jaspers, 1946, p. 622). Y, como es sabido, dicho dinamismo se ha hecho todavía más patente en las últimas décadas, en las que hemos asistido a un notable aumento de la velocidad en el intercambio de bienes e información y a una imparable globalización de las innovaciones económicas, sociales y culturales (Virilio, 1996).

Pues bien, resulta obvio decir que ha sido en todo este contexto de "movilización general" (cuyos inicios suelen datarse en la industrialización de los siglos XVIII y XIX) en el que los patrones de experiencia y conducta que hoy calificamos como psicóticos o esquizofrénicos se han tornado socialmente "visibles" y han sido finalmente "descubiertos" como "nuevas" enfermedades. Desde este punto de vista, y como describió Laing, los esquizofrénicos y otras personas afectadas por trastornos psicóticos encarnan una forma extrema de "disidencia" y se asemejan a un avión que se separa de su escuadrón, sin que, en rigor, pueda decirse si es el avión o todo el escuadrón el que se halla "fuera de ruta" (Laing, 1977, p. 104). Pero, siguiendo con la metáfora y como muestra de forma paradigmática el caso de Don Quijote, parece claro que los "enfermos" puestos al descubierto por el proceso de modernizaciónmovilización no han sido aquellos individuos que han logrado "moverse" en la dirección prescrita por el nuevo orden social, sino, al contrario, aquellos que han permanecido detenidos en su posición de partida. En este sentido, por ejemplo, uno de los elementos que con seguridad ha contribuido a la "visibilidad" de la esquizofrenia ha sido la transición por la cual las antiguas reglas tradicionales de conducta 
han sido progresivamente suplantadas por el régimen de tareas y responsabilidades individuales que distingue actualmente las relaciones interpersonales o laborales. Pues, no en vano, y como sabemos desde los finos análisis de Klaus Conrad (19051961), las primeras crisis y episodios esquizofrénicos tienden a producirse en situaciones que solicitan una "salida a escena" del individuo y se caracterizan por la percepción subjetiva de un alto nivel de responsabilidad (Conrad, 1997).

En cualquier caso, si hay un factor al que podamos otorgar un papel relevante en la aparición histórica de la esquizofrenia éste es, sin duda, la emergencia del sujeto moderno y la progresión de lo que genéricamente podemos definir como la conciencia psicológica. De hecho, puede decirse que la esquizofrenia se convirtió en una condición culturalmente posible en cuanto los seres humanos se vieron forzados a asumir lo que Anthony Giddens ha denominado el "proyecto reflexivo del yo", esto es, a cultivar una interioridad psicológica y a entrar en un intercambio público en torno a la misma (Giddens, 1991).

De este modo, una condición básica para la constitución cultural de la esquizofrenia ha sido la emergencia de ese espacio interior o subjetivo característico del individuo moderno al que sólo él tiene acceso, pero cuyas formas y contenidos participan de un orden simbólico colectivo que los dota de sentido y permite comunicarlos (Taylor, 1996). Por su parte, la formación de esta interioridad ha sido activamente promovida por la marcada escisión entre esfera pública y privada que ha acompañado el despliegue de la Modernidad (McKeon, 2005), y que, como sabemos, ha conducido a una progresiva inserción de la vida familiar, interpersonal y psicológica en los cada vez más extensos dominios de la privacidad. La emergencia de la interioridad moderna ha ido así de la mano de lo que el sociólogo norteamericano Richard Sennett ha denominado la "tiranía de la intimidad" (Sennett, 2002), es decir, de una creciente tendencia a la sinceridad y autenticidad en las relaciones sociales y, por tanto, a una presentación pública del individuo acorde y consonante con su realidad subjetiva. $Y$ no es necesario añadir que esta tendencia ha impregnado hasta el extremo la creación literaria y artística de los últimos siglos, que ha pivotado en gran medida alrededor de la publicación de contenidos privados a través de novelas en primera persona, autobiografías, confesiones y todo tipo de estrategias de desenmascaramiento y revelación del yo o la "verdad interior" (Kundera, 1987).

En estas coordenadas, pues, parece lógico que la locura haya dejado de constituir un acontecimiento exterior, tangible e incluso obsceno (y, en consecuencia, inquietante y potencialmente subversivo) para pasar a ser un cataclismo subjetivo y silencioso que, ante todo, compromete las estructuras de la identidad. Pero, en el caso de la esquizofrenia, la estricta separación entre el ámbito de lo público y lo privado ha facilitado además su constitución por medio de dos factores específicos: por la mayor vulnerabilidad recíproca que han debido soportar entre sí la esfera pública y la privada y, muy especialmente, por la necesidad y la exigencia impuesta a los individuos de "aislar" su mundo interno y comunicar públicamente sus estados subjetivos.

En concreto, esta exigencia implica una serie de operaciones emblemáticas de la conciencia moderna en las que, como hemos visto, el esquizofrénico naufraga de forma característica, poniendo así de manifiesto su alteridad y la esencia misma de su alienación. La primera de ellas concierne la objetivación de los contenidos de conciencia que, como ha subrayado el filósofo canadiense Charles Taylor, se deriva de la sistemática (y necesaria) asunción por parte del sujeto moderno de una posición de tercera persona ante ellos (Taylor, 1996). La segunda se relaciona con la tendencia a la reflexividad y la observación de segundo orden que impregna numerosas facetas de la vida moderna (Giddens, 1990), y que lleva a la conciencia a marchar, por así decirlo, siempre un paso por detrás de sí misma: como en las pinturas de Magritte, no ve el mundo, sino que observa la percepción que hace de él; no percibe las cosas, sino que medita sobre las impresiones que éstas le producen. Y, por último, la tercera tiene que ver con el mismo fenómeno que permitió a Bleuler acuñar su neologismo y teorizar sobre la subjetividad esquizofrénica, a saber, el alto grado de escisión o fragmentación que la conciencia moderna ha de ser capaz de sobrellevar para ejecutar sus operaciones y que, en última instancia, le obliga a verse continuamente confrontada con algo (pensamientos, sensaciones, 
etc.) que no es ella misma, pero que, sin embargo, se aloja en su interior.

La esquizofrenia, en suma, mantiene una relación constitutiva con el reto y la exigencia moderna de instituirse como un sujeto que, a su vez, se objetiva a sí mismo, atiende a sus propias operaciones y fragmenta su conciencia. Y, en ese sentido, puede decirse que no es sino el testimonio más dramático de una cultura que a todos nos convierte en poseedores, informantes y manipuladores de un "yo", y que a todos nos lleva a relacionarnos con el mundo, los demás y nosotros mismos desde el parapeto de una reflexividad que nos aleja de la espontaneidad, la inmediatez y -como deploraban los románticos- quizá también de la vida. Una cultura que, como no podría ser de otra manera, lleva así en su núcleo el germen y el fundamento de su propia alienación.

\section{Extended Summary}

Schizophrenia is not a a mental disorder or an illness like others. Involving and compromising the basic structures of what we associate with nuclear aspects of our culture such as individuality, privacy, identity or agency, schizophrenia is not, as is known, a process suffered on a timely basis, but a condition that pervades the whole existence of its sufferer and confers him a permanent and well defined psychological profile; human beings do not have schizophrenia, they are schizophrenic (or not). But in a broader sense and taking into account the enormous symbolic potential achieved by schizophrenia throughout the twentieth century, it is also not a conventional disease because its very nature seems to say, suggest or even show us something important and essential with respect to the "spirit of our times". In fact, even today we see in it a radical experience that, as our times, refers to insecurity and fear, to anomy and unorthodoxy, to the misterious and uncanny, and leads to the destruction of interpersonal links, to a break with history and tradition and to an abandonment of the common territory of shared language and meanings. Actually, it is difficult to find descriptions or diagnoses of our times and culture -that what we use to call Modernity- that do not allow to draw close parallels and analogies with the schizophrenic condition.

Starting from the consideration of this constituive affinity, this article proposes a panoramic assessment of the history of schizophrenia that tries to unravel not only its configuration as one of the most emblematic clinical pictures of contemporary psychiatry, but also as a severe disturbance of self-consciousness, bodily experience and social life which is rooted in the potential for alienation released by the structures of modern subjectivity. For this purpose, we describe first the process leading to the description of the clinical concept of schizophrenia in the classic works of Emil Kraepelin, Eugen Bleuler and Kurt Schneider. Later, and as a counterpoint to this external or objective characterization, we present some major guidelines concerning the reconstruction of schizophrenic experience undertaken by phenomenological psychopathology. And, finally, we provide a brief discussion of the major factors and areas involved in the constitution of schizophrenia as a disorder which is closely related to the gradual deployment of our current social and cultural order.

As is known, the history of the concept of schizophrenia has its most famous and well-defined start in the description of the so-called Dementia pracox by German psychiatrist Emil Kraepelin (18561926). Kraepelin gathered under the heading of "deterioration processes" (Verblödungsprocesse) a set of clinical states formed by Dementia praecox, Katatonia and Dementia paranoides. These three forms, grouped in the fifth edition of his Lehrbuch (1896), were finally considered to be a single disease-Dementia praecox- from the sixth edition of 1899 on. As is known, Kraepelin established then two major groups of endogenous psychoses: manic-depressive psychosis, curable, and Dementia praecox, incurable. Subsequently, he completed this classification by distinguishing two evolutive forms of Dementia praecox: the progressive one, leading to a permanent impairment, and the intermittent one, not leading to an irreversible deficit. Although the grouping in a single disease of the 
paranoid forms with hebephrenia and catatonia seemed to be somewhat forced from a theoretical point of view and was heavily criticized by French psychiatrists, Kraepelin thought that this set of clinical conditions actually had the common particularity of leading to states of a characteristic mental deterioration. And thus, he described a series of deficit symptoms -affective deterioration (gemüthliche Verblödung), apathy, indifference, disorganization of thought and speech, incoherence (Zerfahrenheit) and others- as the nuclear manifestations of Dementia praecox.

For his part, Swiss psychiatrist Eugen Bleuler (1857-1940) published in 1911 his Dementia praecox oder Gruppe der Schizophrenien, where he offered a new description of the disease from the point of view of what he considered to be its most prominent psychopathological trait: the splitting of personality. As is known, this new perspective led Bleuler to introduce the neologism "schizophrenia" (literally "split mind") to designate the entity isolated by Kraepelin, but his proposal did not merely suggested a terminological variation. On the contrary, it stemmed from a new style of seeing insanity and the insane according to which psychopathological interpretation was of central importance and symptomatology and evolution should pass into the background. So, Bleuler considered that the schizophrenic process was defined by a particular form of "splitting" (Spaltung) of the mental functions resulting from the action of independent "complexes" or chains of representations and emotions that ended up by dominating the entire personality and compromising its unity. Inspired by the psychometric studies of his young assistant Carl Gustav Jung (18751961) on the disorders affecting the associations of ideas and the early Freudian explanations on the mechanisms governing unconscious formations, Bleuler thought that schizophrenia was due to a primary weakness in the sphere of associations that prevented a proper integration of the contents of consciousness, thus leading to the disorganization of psychic activity which was characteristic of the disease.

With this background, the work of German psychiatrists diversified in the subsequent decades between the comprehensive analysis of schizo- phrenic psychopathology and the clinical identification of those signs and symptoms that could allow a more reliable diagnosis. In this sense, the author who distinguished himself in a more remarkable way was undoubtedly Kurt Schneider (1887-1967), who in 1946 took in charge the prestigious University Chair of Heidelberg and provided an extremely influential characterization of schizophrenic symptoms. Bypassing any reference to evolution or prognosis and thinking that pure clinical observation (i.e. devoid of any theoretical considerations) was sufficient to establish the diagnosis, Schneider identified a series of psychotic experiences which he called of "first rank" due to their usefulness to detect the presence of schizophrenia. But most of these first-rank symptoms (audible thoughts, voices heard arguing, voices heard commenting on one's actions, bodily experiences of influence, thought withdrawal, thought insertion, etc.) shared a common phenomenological trait: the loss of ego-boundaries and, in short, an experience of one's mental activity (language, thoughts, feelings, intentions, etc.) as alien or imposed. And thus, Schneider's contribution provided the third major component of the current clinical concept of schizophrenia, adding to Kraepelinian deterioration and Bleulerian disorganization the so called "disorders of self-experience" (Ich-Störungen) as the key feature of the condition.

Concerning the analysis and reconstruction of the inner world and subejctive experiences of schizophrenics, it must be noted that they represent some of the most significants achievements of phenomenological psychopathology. With the double goal of providing a global and empathetic understanding of the clinical picture and describing its experience "from the inside", the categories and insights delivered from this tradition (dating back to the seminal work of French psychiatrist Eugène Minkowski in the 1920s) have resulted in a characterization of the specificity of the schizophrenic alienation which not only turns intelligible its different clinical manifestations, but also allows to understand and place its major features in a broader historical and cultural context. In general terms, the phenomenological analysis has focused on the account of three realms of phenomena that concern, respectively, social life, 
bodily experiences and self-consciousness. And thus, the phenomenological point of view has allowed to outline the contours of the schizophrenic alienation in terms which stand quite far from the traditional view of madness as an "incomprehensible" regression or claudication of reason. Then, in fact, by paying attention to autism, to the obliteration of "common sense" or to the disturbances of selfhood and self-consciousness, the schizophrenic world as uncovered by this perspective seems rather to be a highly complex world, solitary, hyperrational, cold and perfectly adynamic and inert. And, in this sense, it is not surprising that schizophrenic experience has often been equated with modern consciousness itself and its positivist science, as both tend to disembody the world and to deprive it from every sign of vitality or transcendence; as both move the subject beyond a shared order of meaning; and as both tend to reduce all phenomena to their mere presentation and transform life itself into a raw and hopeless machinery.

These analogies between schizophrenia and modern consciousness lead us finally to raise some considerations on their constitutive affinity, and more particularly, on the main factors and areas involved in the emergence of schizophrenia as an event which is closely related to the development of modern culture. Thus, and in the same way that schizophrenia does not use to affect individuals before adolescence -since only in this period of life seem to emerge the necessary psychic conditions-, on a social and cultural level a number of circumstances had to be present in order to allow that a previously indefinite pattern of otherness and subjectivity could be conceived and identified as "schizophrenic". And given that, as we have seen, schizophrenia typically compromises cardinal elements of our social and cultural order such as the attribution of psychological agency, the construction of identity, the sequential performance of social roles or the public presentation of the self, it is not difficult to isolate some factors that converge on two basic points, namely, the dramatic social and psychological mobilization of the individuals induced by the process of modernization and the gradual deployment of psychological consciousness and the reflexive individuality which is characteristic of our culture.

\section{Referencias}

Álvarez, J.M. (1996). La invención de las parafrenias. Revista de la Asociación Española de Neuropsiquiatría, 16 (57), 75-85.

Álvarez, J.M. (2008). La invención de las enfermedades mentales. Madrid: Gredos.

Álvarez, J.M. y Colina, F. (2007). Las voces y su historia: sobre el nacimiento de la esquizofrenia, Átopos, 6, 4-12.

Andreasen, N. (1997). The evolving concept of schizophrenia from Kraepelin to present and future. Schizophrenia Research, 28, 105-109.

Andreasen, N. y Olsen, S. (1982). Negative vs. positive schizophrenia: Definition and validation. Archives of General Psychiatry, 39, 789794.

Angst, J. (2002). Historical aspects of the dichotomy between manic-depressive disorders and schizophrenia. Schizophrenia Research, 57, 5-13.

Arieti, S. (1965). Interpretación de la esquizofrenia. Barcelona: Labor.

Baruk, H. (1974). La révision de la schizophrénie. Annales médico-psychologiques, 2, 261-277.

Beer, D. (1996). The endogenous psychoses: a conceptual history. History of Psychiatry, 7, 1-29.

Bercherie, P. (1986). Los fundamentos de la clínica. Historia y estructura del saber psiquiátrico. Buenos Aires: Manantial.

Berger, P. y Luckmann, T. (1997). Modernidad, pluralismo y crisis de sentido. Barcelona: Paidós.

Blankenburg, W. (1971). Der Verlust der natürlichen Selbstverständlichkeit. Stuttgart: Enke.

Bleuler, E. (1926). Zur Unterscheidung des Physiogenen und des Psychogenen bei der Schizophrenie. Allgemeine Zeitschrift für Psychiatrie, 84, 22-37.

Bleuler, E. (1961). Demencia precoz. El grupo de las esquizofrenias. Buenos Aires: Hormé.

Bleuler, E. (1969). Afectividad, sugestibilidad, paranoia. Madrid: Morata.

Bleuler, E. (1996). La esquizofrenia (1926). Revista de la Asociación Española de Neuropsiquiatría, 60, 663-676.

Bovet, P. y Seywert, F. (1995). La schizophrénie et son spectre. Une perspective bleulérienne. L'Information psychiatrique, 71, 447-458. 
Boyle, M. (1990). Schizophrenia: a scientific delusion? Londres: Routledge.

Brockington, I. (1992). Schizophrenia: Yesterday's concept. European Psychiatry, 7, 213-220.

Chandrasena, R.D. (1983). Phenomenology and nosology of schizophrenia: historical review. Psychiatric Journal of the University of Ottawa, 8 (2), 17-24.

Colodrón, A. (1983). Las esquizofrenias, Madrid, Siglo XXI.

Colodrón, A. (2002). La condición esquizofrénica. Madrid: Triacastela.

Conrad, K. (1997). La esquizofrenia incipiente. Madrid: Triacastela.

Crow, T.J. (1985). The two-syndrome concept: Origins and current status. Schizophrenia Bulletin, $11,471-486$.

Ellenberger, H. (1976). El descubrimiento del inconsciente. Historia y evolución de la psiquiatría dinámica. Madrid: Gredos.

Estroff, S.E. (1989). Self, identity, and subjective experiences of schizophrenia: in search of the subject. Schizophrenia Bulletin, 15, 189-196.

Fabrega, H. (1989). The self and schizophrenia: a cultural perspective. Schizophrenia Bulletin, 15, 277-290.

Fraguas, D. y Breathnach, C.S. (2009). Problems with retrospective studies of the presence of schizophrenia. History of Psychiatry, 20, 61-71.

Fuchs, T. (1995). Coenästhesie: Zur Geschichte des Gemeingefühls. Zeitschrift für klinische Psychologie, Psychopathologie und Psychotherapie, 43, 103-112.

Fuchs, T. (2005). Corporealized and Disembodied Minds: A Phenomenological View of the Body in Melancholia and Schizophrenia. Philosophy, Psychiatry \& Psychology, 12, 95-107.

Garrabé, J. (1996). La noche oscura del ser. Una historia de la esquizofrenia. México: FCE.

Giddens, A. (1990). The Consequences of Modernity. Oxford: Polity Press.

Giddens, A. (1991). Modernity and Self-Identity: Self and Society in the Late Modern Age. Stanford CA: Stanford University Press.

Gruhle, H.W. (1913). Bleulers Schizophrenie und Kraepelins Dementia Precox. Zeitschrift für die gesamte Neurologie Psychiatrie, 17, 114-133.
Hoenig, J. (1983). The concept of schizophrenia. Kraepelin-Bleuler-Schneider. British Journal of Psychiatry, 142, 547-556.

Hoff, P. (1994). Emil Kraepelin und die Psychiatrie als klinische Wissenschaft. Berlin: Springer.

Huertas, R. (1987). Locura y degeneración. Psiquiatría y sociedad en el positivismo francés. Madrid: CSIC.

Jaspers, K. (1946). Allgemeine Psychopathologie. Berlín: Springer.

Jung, C.G. (1906). Diagnostische Assoziationsstudien: Beiträge zur experimentellen Psychopathologie. Leipzig: J.A. Barth.

Kraepelin, E. (1899). Psychiatrie. Ein Lehrbuch für Studierende und Ärtze, $6^{\mathrm{a}}$ ed. Leipzig: J.A. Barth.

Kraus, A. (2001). Phenomenological-Anthropological Psychiatry. En F. Henn, N. Sartorius, H. Helmchen y H. Lauter (Eds.) Contemporary Psychiatry, Volume 1 (340-55). Berlín: Springer.

Kundera, M. (1987). El arte de la novela. Barcelona: Tusquets.

Laing, R.D. (1964). El yo dividido. México: FCE.

Laing, R.D. (1977). La política de la experiencia. Barcelona: Crítica.

Lantéri-Laura, G. (2000). Ensayo sobre los paradigmas de la psiquiatría moderna. Madrid: Triacastela.

LeBlanc, A. (2001). The origins of the concept of dissociation: Paul Janet, his nephew Pierre, and the problem of post-hypnotic suggestion. History of Science, 39, 57-69.

Leferink, K. (1997). Sympathie mit der Schizophrenie. Die Moderne und ihre psychische Krankheit. En: M. Zaumseil y K. Leferink (Eds.) Schizophrenie der Moderne, Modernisierung der Schizophrenie. Lebensalltag, Identität und soziale Beziehungen von psychisch Kranken in der Grossstadt (27-81). Bonn: Edition Das Narrenschiff im Psychiatrie-Verlag.

Mahieu, E.L. (2004). A propósito de Morel y la démence précoce. Vertex, 15, 73-75.

Maj, M. (1998). Critique of the DSM-IV operational diagnostic criteria for schizophrenia, British Journal of Psychiatry, 172, 458-460.

McKeon, M. (2005). The Secret History of Domesticity: Public, private, and the division of knowledge. Baltimore MD: The John Hopkins 
University Press.

Minkowski, E. (2000). La esquizofrenia. Psicopatología de los esquizoides y los esquizofrénicos. México: FCE.

Morel, B.A. (1852-53). Études cliniques. Traité théorique et pratique des maladies mentales. Nancy: Grimblot.

Morel, B.A. (1860). Traité des maladies mentales. Paris: Baillière.

Peralta, V. y Cuesta, M.J. (2000). Clinical models of schizophrenia: a critical approach to competing conceptions. Psychopathology, 33, 252-258.

Polanyi, M. (1967). The Tacit Dimension. Chicago: University of Chicago Press.

Rümke, H.C. (1990). The nuclear symptom of schizophrenia and the "praecox feeling". History of Psychiatry, 1, 331-341.

Sass, L.A. (1992). Madness and Modernism: Insanity in the Light of Modern Art, Literature and Thought. Nueva York: Basic Books.

Sass, L.A. y Parnas, J. (2003). Schizophrenia, consciousness, and the self. Schizophrenia Bulletin, 29, 427-444.
Schneider, K. (1997). Psicopatología clínica. Madrid: Triacastela.

Séglas, J. (1900). La démence paranoïde. Annales médico-psychologiques, 12, 232-246.

Sennett, R. (2002). El declive del hombre público. Barcelona: Península.

Serbski, V. (1903-04). Contribution à l'étude de la démence précoce Annales médico-psychologique, 18, 379-388; 19, 19-34; 19, 188-203.

Stanghellini, G. (2004). Disembodied Spirits and Deanimated Bodies. The psychopathology of common-sense. Oxford: OUP.

Stotz-Ingenlath, G. (2000). Epistemological aspects of Eugen Bleuler's conception of schizophrenia in 1911. Medicine, Health Care and Philosophy, 3, 153-159.

Taylor, Ch. (1996). Fuentes del yo: La construcción de la identidad moderna. Barcelona: Paidós.

Virilio, P. (1996). El arte del motor. Aceleración y realidad virtual. Buenos Aires: Manantial.

Wilmanns, K. (1932). Die Schizophrenie. En O. Bunke (Ed.). Handbuch der Geisteskrankheiten, Teil 5, Band 9. Berlín: Springer.

Manuscrito recibido: 31/08/2010

Revisión recibida: 13/09/2010

Manuscrito aceptado: 15/09/2010 